Published in "Blood 136(12): 1375-1376, 2020"

which should be cited to refer to this work.

\section{Erythroblasts provide a home for gametocytes}

Pierre-Yves Mantel | University of Fribourg

In this issue of Blood, Neveu and colleagues shed new light on the development of Plasmodium falciparum gametocytes in the bone marrow. They demonstrate that gametocytes invade and mature inside erythroblasts in the erythroblastic islands, whereas parasite infection and extracellular vesicles secreted by parasites delay the maturation of the erythroblast to allow the gametocyte maturation to coincide with release of their host cell from the bone marrow. ${ }^{1}$

The most severe forms of malaria are caused by the parasites $P$ falciparum, which requires 2 hosts to fulfill its life cycle. While in the human host, the parasites cause most of their burden during the blood stage. The asexual replication that occurs in the red blood cells lasts 48 hours, during which time the parasites undergo a complex process of maturation and remodeling of the host cells. At the end of the 48-hour cycle, the parasites

egress from the infected red blood cells and invade uninfected red blood cells, and the cycle will then repeat.

During the asexual cycle, a small proportion of parasites commits to produce a sexual progeny to initiate the sexual cycle. Gametocytes go through 5 different stages of maturation that take $\sim 10$ days to complete. The gametocyte is the only form of the parasites that can be transmitted to

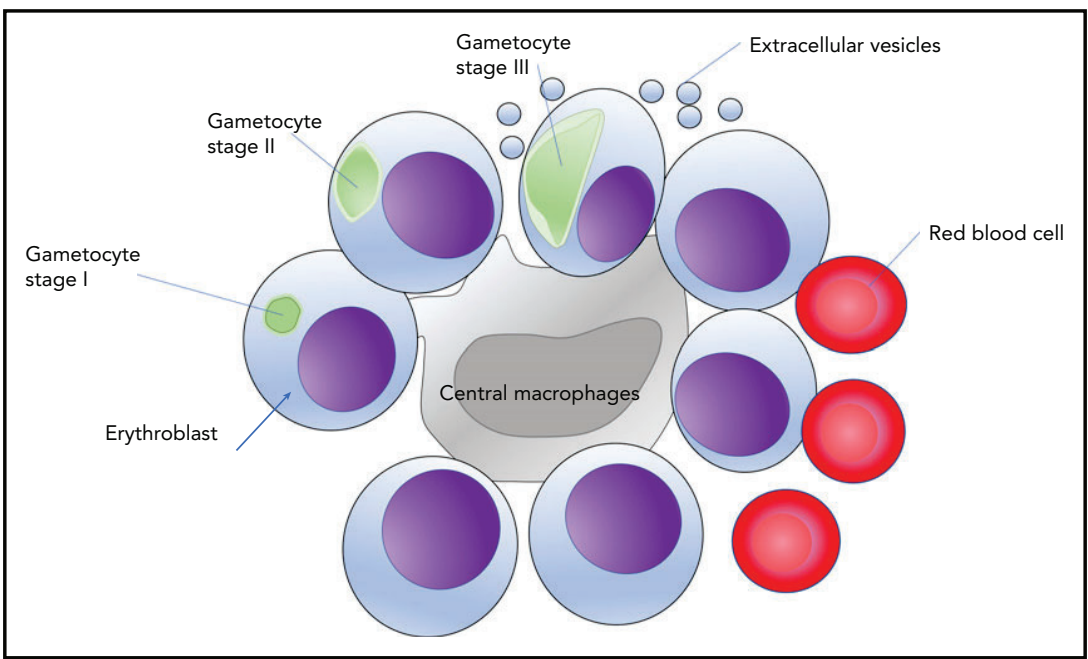

Gametocytes invade, differentiate, and mature in erythroblasts. Immature gametocytes from stages I to IV can be observed inside erythroblasts. This strategy allows the gametocytes to grow inside the bone marrow in the erythroblastic islands surrounding a central macrophage. the mosquito. Therefore, the transmission stage constitutes a bottleneck for the parasites and may represent an important target for drug or vaccine with the aim to eliminate malaria. Mature gametocytes can circulate in the peripheral blood inside mature red blood cells until taken up by a mosquito during a blood meal and therefore can be observed in blood smears, whereas immature gametocytes preferentially accumulate and develop in the bone marrow parenchyma around the erythroblastic islands. Immature gametocytes from stages I to IV are not found in the blood circulation. It was observed $>100$ years ago that they are preferentially hiding in the bone marrow. ${ }^{2}$ This was further confirmed by several case studies in which immature gametocytes were observed in erythroblastic islands. ${ }^{3}$ However, only recently more detailed histological and molecular studies confirmed their sequestration in the bone marrow. ${ }^{4,5}$

The bone marrow might provide an ideal site for the parasites to develop while hiding from the immune system and avoiding clearance by the spleen. However, the mechanisms involved in the sequestration are ill defined, and several hypotheses exist. Immature gametocyte maintenance in this microenvironment may be dependent on the adhesion to nonendothelial bone marrow cells, such as the mesenchymal cells. ${ }^{6}$ Another hypothesis not mutually exclusive is that the increased rigidity of immature gametocytes may contribute to their sequestration locally by mechanical retention. ${ }^{7}$ Another unexplored possibility is that gametocytes directly infect cells in the erythroblastic islands.

In this issue of Blood, Neveu and colleagues investigated the cellular interactions of the gametocytes with the cellular compartment of the hematopoietic niche. More specifically, they hypothesized that gametocytes call erythroblasts home. To address this hypothesis, the authors used human primary erythroblasts derived from granulocyte colony-stimulating factor-mobilized peripheral blood. Then the erythroblasts 
were infected by synchronized purified schizonts. The parasites were able to invade and grow inside erythroblasts. Erythroblasts are nucleated cell precursors of mature red blood cells and differentiate in the bone marrow, in particular, in the erythroblast islands. Although it is known that the asexual parasites can develop in nucleated cells, the fate of gametocytes was never addressed. Here, not only gametocytes were observed inside erythroblasts but also they were able to mature inside this new home. Interestingly, the gametocytes upon activation by temperature drop were able to produce gametes, suggesting that the maturing gametocytes are functional and might be picked up by mosquitoes. Furthermore, the discovery was validated in vivo, by analyzing the bone marrow of a $P$ falciparum-infected patient. Gametocytes were identified by staining with an antibody against the plasmodial protein Pf11.1 and found in nucleated cells that stained positive for red blood cell protein glycophorin A, in other words, erythroblasts (see figure).

Surprisingly, Neveu and colleagues noticed that the maturation of the parasiteinfected erythroblasts was slowed down. Further investigations demonstrated that infections in combination with parasitederived extracellular vesicles delayed the erythroid differentiation. The delay in differentiation was correlated with oxidative stress. Both infection and extracellular vesicles were able to directly induce oxidative stress in erythroblasts.

These findings raise several interesting questions: anemia in malaria patients is caused not only by rupture of infected red blood cells but also by the inhibition of hematopoiesis. One can wonder whether the gametocyte sequestration contributes to blockage of hematopoiesis.

Extracellular vesicles are small vesicles secreted by infected red blood cells. Besides potent immunomodulatory properties, extracellular vesicles have a role in cellular communication by transferring signaling cargoes between a donor cell to an acceptor cell.8,9 Do extracellular vesicles transfer functional cargoes into erythroblasts to regulate their function?

Finally, after invasion, the parasites remodel the host cells by exporting proteins into the cytosol. The authors raise the question that gametocytes might hijack the host cells by secreting proteins with nuclear localization signal that could interfere with gene regulation in the host cells.

Altogether, this work provides solid evidence for a new mechanism of gametocyte retention in the bone marrow by direct infection of erythroblasts.

Conflict-of-interest disclosure: The author declares no competing financial interests.

\section{REFERENCES}

1. Neveu G, Richard C, Dupuy F, et al. Plasmodium falciparum sexual parasites develop in human erythroblasts and affect erythropoiesis. Blood. 2020;136(12):1381-1393.

2. Marchiafava E, Bignami A. Sulle Febbri Estivo Aumnali. E. Loescher; 1894.

3. Farfour E, Charlotte F, Settegrana C, Miyara M, Buffet $P$. The extravascular compartment of the bone marrow: a niche for Plasmodium falciparum gametocyte maturation? Malar J. 2012; 11(1):285.

4. Aguilar R, Magallon-Tejada A, Achtman AH, et al. Molecular evidence for the localization of Plasmodium falciparum immature gametocytes in bone marrow. Blood. 2014;123(7):959-966.
5. Joice R, Nilsson SK, Montgomery J, et al. Plasmodium falciparum transmission stages accumulate in the human bone marrow. Sci Transl Med. 2014;6(244):244re5.

6. Messina V, Valtieri M, Rubio $M$, et al. Gametocytes of the malaria parasite Plasmo dium falciparum interact with and stimulate bone marrow mesenchymal cells to secrete angiogenetic factors. Front Cell Infect Microbiol. 2018;8:50.

7. Tibúrcio M, Niang M, Deplaine G, et al. A switch in infected erythrocyte deformability at the maturation and blood circulation of Plasmodium falciparum transmission stages. Blood. 2012;119(24):e172-e180.

8. Mantel PY, Hoang AN, Goldowitz I, et al. Malaria-infected erythrocyte-derived microvesicles mediate cellular communication within the parasite population and with the host immune system. Cell Host Microbe. 2013;13(5): 521-534.

9. Mantel PY, Hjelmqvist D, Walch $M$, et al. Infected erythrocyte-derived extracellular vesicles alter vascular function via regulatory Ago2miRNA complexes in malaria. Nat Commun. 2016;7(1):12727. 